\title{
Experimental Suppression of TMS-EEG Sensory Potentials
}

\author{
Jessica M. Ross ${ }^{1,2}$, Manjima Sarkar², Corey J. Keller ${ }^{2,1, \delta}$ \\ ${ }^{1}$ Veterans Affairs Palo Alto Healthcare System, and the Sierra Pacific Mental Illness, \\ Research, Education, and Clinical Center (MIRECC), Palo Alto, CA, 94394, USA \\ ${ }^{2}$ Department of Psychiatry and Behavioral Sciences, \\ Stanford University Medical Center, Stanford, CA, 94305, USA
}

${ }^{\delta}$ Correspondence:

Corey Keller, MD, PhD

Stanford University

Department of Psychiatry and Behavioral Sciences

401 Quarry Road

Stanford, CA 94305-5797

Email: ckeller1@stanford.edu

Phone: +18025786292

Number of pages: 23

Number of figures: 3

Number of tables: 0

Abstract word count (250): 248

Total word count: 5064 with citations

\section{Running title: Reducing TMS-EEG Sensory Potentials}

Keywords: Transcranial magnetic stimulation (TMS), electroencephalogram (EEG), evoked potentials, Vertex Potential (VP), Auditory Evoked Potential (AEP)

Funding: This work was supported by R01MH129018 and the Burroughs Wellcome Fund Career Award for Medical Scientists. 


\begin{abstract}
Background: The sensory experience of transcranial magnetic stimulation (TMS) evokes cortical responses measured in EEG that confound interpretation of TMS-evoked potentials (TEPs). Methods for sensory masking have been proposed to minimize sensory contributions to the TEP, but the most effective combination for suprathreshold TMS to dorsolateral prefrontal cortex (dlPFC) is unknown.
\end{abstract}

Objective: We applied sensory suppression techniques and quantified electrophysiology and perception from suprathreshold dIPFC TMS to identify the best combination to minimize the sensory TEP.

Methods: In 21 healthy adults, we applied single pulse TMS at $120 \%$ resting motor threshold (rMT) to the left dlPFC and compared EEG vertex N100-P200 and perception. Conditions included three protocols: No masking (no auditory masking, no foam, jittered inter-stimulus interval (ISI)), Standard masking (auditory noise, foam, jittered ISI), and our ATTENUATE protocol (auditory noise, foam, over-the-ear protection, unjittered ISI).

Results: ATTENUATE reduced vertex N100-P200 by 56\%, "click" loudness perception by $50 \%$, and scalp sensation by $36 \%$. We show that sensory prediction, induced with predictable ISI, has a suppressive effect on vertex N100-P200, and that combining standard suppression protocols with sensory prediction provides the best N100-P200 suppression. ATTENUATE was more effective than Standard masking, which only reduced vertex N100-P200 by $22 \%$, loudness by $27 \%$, and scalp sensation by $24 \%$.

Conclusions: We introduce a sensory suppression protocol superior to Standard masking and demonstrate that using an unjittered ISI can contribute to minimizing sensory confounds. ATTENUATE provides superior sensory suppression to increase TEP signalto-noise and contributes to a growing understanding of TMS-EEG sensory neuroscience.

\title{
Highlights:
}

- ATTENUATE is a novel sensory suppression protocol for suprathreshold dIPFC TMS

- ATTENUATE is superior to standard masking for minimizing sensory confounds

- ATTENUATE reduced vertex N100-P200 by 56\% with no effect on the early TEP

- ATTENUATE reduced "click" loudness rating by $50 \%$ and scalp sensation by $36 \%$

- Individual modifications are not sufficient to reduce vertex N100-P200 or perception 


\section{Introduction}

Transcranial magnetic stimulation (TMS) is a powerful non-invasive tool for stimulating brain networks [1-3] and has proven useful for the neurophysiological characterization and treatment of neurological and psychiatric disorders [4-8]. Neural changes caused by TMS are measurable and quantifiable using electroencephalography (EEG) [9-13]. For instance, an averaged single pulse TMS-evoked EEG potential (TEP) can be used to characterize local and network excitability as well as plasticity following repetitive TMS protocols [10,14-16]. Gaining a better understanding of and utilizing TMS-induced EEG changes is critical for targeted and personalized circuit manipulation for robust clinical use.

While TEPs are a promising measure of TMS-evoked neural activity, it has become evident that off-target sensory effects of single TMS pulses can severely confound the interpretation of the TEP [17-22]. These off-target effects include sensory potentials that are peripherally evoked due to the multisensory nature of TMS [23]. Although the TEP is reproducible $[9,24]$ and has been shown to reflect localized TMS-evoked activity at the earliest latencies after the pulse (up to approximately 60-80ms) [11,16,20,23,25-27], there is accumulating evidence that the later TEP (>80ms) is contaminated by off-target sensory potentials [18-20]. One such component of the later TEP is an evoked response [28] induced from the sound of TMS (referred to as the auditory evoked potential, AEP) and not specific to the site of stimulation [17,23]. The greatest amplitude and most robustly measured subcomponents of this sensory potential occur at the vertex at 100 and $200 \mathrm{~ms}$ with an accompanying smaller potential at $\sim 50 \mathrm{~ms}[22,25,29-33]$. These vertex potentials are described as an N100-P200 complex, which overlaps with all but the earliest TEP components. In summary, sensory potentials in the TEP remain a significant confound to the direct effects of TMS and minimization or removal is necessary to improve interpretability.

Experimental modifications have been proposed to suppress the sensory vertex N100P200, but the most effective combination for suprathreshold TMS is unknown. This is particularly true for targeting the dlPFC, the primary treatment location for many neuropsychiatric disorders [34-39]. Here, we focus on the following experimental modifications: auditory masking, a foam separator between the coil and the scalp, and predictably spacing TMS pulses. A common sensory masking protocol is to pair earplugs and/or auditory noise masking [2,19], a foam separator, and a jittered inter-stimulus interval (ISI) (hereafter called Standard masking). Recent evidence for effective standard masking is promising for subthreshold TMS to primary motor cortex $(90 \%$ of the resting motor threshold (rMT) [2,19]). However, it has also been shown that these methods often do not fully suppress the sensory vertex N100-P200 [17,18,40-44], particularly for higher intensity protocols $[17,40]$. Rocchi et al. [19] used over-the-ear protection in addition to noise masking to further minimize vertex N100-P200, with positive results for subthreshold M1 stimulation. However, how over-the-ear protection performs for higher stimulation intensities and non-M1 targets is unknown. The use of foam padding between the coil and scalp is thought to suppress the vertex N100-P200 by reducing bone conduction of the sound [41]. However, it is unclear what type or thickness of foam should 
be used. In addition, there is no consensus regarding how to adjust stimulation intensity to account for higher coil to cortex distance when foam is added. Modifying the inter-stimulus interval (ISI) timing changes the predictability of TMS pulses, which can have an effect on MEP amplitude [45,46]. However, whether more predictable TMS timing results in a similar attenuation of the TEP is unknown. In summary, a thorough investigation into the optimal experimental methodology to suppress sensory vertex N100-P200 following suprathreshold TMS to the clinically significant dlPFC is necessary.

In this study, we develop an optimal combination of experimental modifications that maximally reduce the vertex N100-P200 complex and sensory perception following suprathreshold single pulse TMS to the dIPFC. In a sample of 21 typically healthy adults, we compared the effects of three masking protocols -: No masking, Standard masking, and a novel procedure - on the vertex N100-P200 and on perception of the TMS loudness, scalp sensation, and pain. We hypothesized that our novel combination of experimental procedures, with the addition of further sound dampening and modification of TMS timing, would best suppress the non-specific sensory component of the TEP. This work contributes to a growing understanding of TMS-EEG sensory neuroscience, and the novel protocol has the potential to enhance interpretability of TMS-EEG studies.

\section{Methods}

\subsection{Participants and Study Design}

All data were collected at Stanford University under an approved institutional review board protocol after participants gave their written informed consent. Participants $(\mathrm{N}=21)$ were 19-64 years old (44.0 mean +/- 14.58 SD) and without current psychiatric or neurological diagnoses. A wide age range was chosen so as not to constrain findings to any a priori group. Supplementary table S1 includes demographic information for all subjects. For each participant, the experiment was conducted on a single day. The experiment was split into multiple single pulse TMS-EEG blocks. Each block consisted of 80 individual single pulse TMS trials applied to the left dlPFC. 80 trials were chosen as they provided high test-retest reliability of the N100 and P200 [9]. TMS-evoked potentials (TEPs) and perceptual scores were quantified, as described below and in schematic in Figure 1A.

\subsection{Transcranial Magnetic Stimulation}

TMS was performed with a MagPro X100 stimulator (MagVenture, Denmark) and a MagVenture Cool B65 figure-of-eight coil (MagVenture, Denmark). The motor hotspot for the right first dorsal interosseous (FDI) was determined by delivering single TMS pulses to the left motor cortex. Resting motor threshold (rMT) was obtained once at the beginning of the experiment and defined as the intensity that produced a visible twitch in relaxed FDI in $\geq 5 / 10$ stimulations [47,48]. Neuronavigation (Localite TMS Navigator MR-less system, Alpharetta, GA) was utilized to determine the left dorsolateral prefrontal cortex (dlPFC) location on a standard Montreal Neurological Institute (MNI) brain map, fitted to individual participants' heads based on scalp measurements. The left dlPFC site (MNI -38, 22, 38) was used to target the fronto-parietal control network [49]. TMS coil 
angle was placed at the angle between 0 and 90 degrees [50-54] that most minimized discomfort and pain for each subject $(\mathrm{M}=52$ degrees, $\mathrm{SD}=27)$. Supplementary table S1 includes the optimal angle for each subject.

To identify the set of procedures that maximally reduces the vertex N100-P200, we tested our novel combination of experimental procedures, which we refer to as ATTENUATE (Auditory: noise masking, Timing: unjittered ISI, Tactile: foam, and over-the-Ear

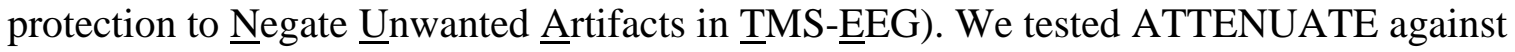
a common Standard masking procedure (auditory noise, foam, jittered ISI) and No masking (no auditory noise, no foam, jittered ISI). See Figure 1B for schematics of the three masking procedures. As noted in the Introduction, it is becoming increasingly common in TMSEEG studies to employ the Standard masking protocol that uses foam and a "click" frequency auditory masking noise $[17,19]$.

In follow-up contrasts we quantify the effectiveness of auditory masking (auditory noise with and without over-the-ear protection), foam, and ISI timing modifications alone (i.e. when each of the other factors is held constant). Figure 3A,H,O depict auditory, foam, and ISI timing conditions, respectively. Conditions were presented in a pseudorandomized order. All conditions were collected in each subject unless the experiment ended early due to time constraints. Supplementary tables S2-S3 reflect for each subject the conditions performed.

Each TMS-EEG condition consisted of 80 single pulses (biphasic pulses at $280 \mu$ s pulse width) at an intensity of $120 \% \mathrm{rMT}$. Stimulator recharge was delayed to $500 \mathrm{~ms}$ to prevent recharge artifact from affecting EEG in the time period of interest [55]. Participants were instructed to keep their eyes open and gaze relaxed throughout each run. For conditions using auditory noise, the noise sound matched the frequency of the TMS click [11] and was delivered with earplug earbuds (Elgin USA Ruckus Earplug Earbuds, NRR 25 dB, Arlington, Texas) at the maximum volume comfortable for each participant. In conditions using over-the-ear protection, to further dampen the TMS "click" sound before reaching the ear canal, we used over-the-ear noise-reducing foam-filled earmuffs (3M Ear Peltor Optime 105 behind-the-head earmuffs, NRR 29 dB, Maplewood, Minnesota). In conditions without auditory noise, earplug earbuds were still kept in the ear canals but no noise was played. In conditions requiring foam, a thin $(0.5 \mathrm{~cm})$ foam pad was attached to the TMS coil, and rMT was redetermined using this foam to accurately deliver a TMS intensity at $120 \%$ rMT while accounting for the increase in coil to scalp distance (see [23] for effects of separator and of distance to scalp on amplitude of vertex N100-P200). Supplementary table S1 includes all rMTs, with and without foam, for all subjects. To determine whether predictability of TMS pulses can attenuate sensory components in the TEP, we compared jittered ISI ( $2 \pm 1 \mathrm{~s}$ jitter) and unjittered ISI ( $2 \mathrm{~s})$ protocols.

\subsection{Electroencephalography}

64-channel EEG data were obtained using a BrainVision actiCHamp Plus amplifiers (5 $\mathrm{kHz}$ sampling rate), with ActiCAP slim active electrodes in an extended 10-20 system montage (actiCHamp, Brain Products GmbH, Munich, Germany). EEG data were online 
referenced to Afz, recorded using BrainVision Recorder software v1.24.0001 (Brain Products $\mathrm{GmbH}$, Germany). Impedances were maintained below $5 \mathrm{k} \Omega$.

\subsubsection{Preprocessing of TEPs}

All EEG preprocessing and analyses were performed in MATLAB R2021a (Mathworks, Natick, MA, USA) using the EEGLAB v2021.1 toolbox [56] and custom scripts. Removal of artifactual EEG data was performed using a custom preprocessing pipeline, as is most common [57], but followed most closely with Ross et al. [40] (steps prior to sensory removal), TMSEEG [58], and TESA [59]. Due to a marked impact of preprocessing pipelines on the TEP, as reported in [57], we took a conservative approach in all steps that required human judgement (with minimal data deletion) and describe all preprocessing steps used in detail with justification for each choice and supporting literature.

All details of EEG data cleaning can be found in Supplementary section S1.1.

\subsubsection{Quantification of TEPs}

For time window and region of interest (ROI) selection, and calculation of global mean field power (GMFP) and local mean field power (LMFP), see Supplementary section S1.2 and Figures S2-5. To compare vertex N100-P200 across experimental conditions, TEPs were generated as averages over the vertex ROI: FC1, FCz, FC2, C1, Cz, C2 (Figure S5C for ROI). LMFP was calculated for the ROI and the area under the curve (AUC) of the LMFP was quantified for the appropriate time windows. Supporting that our time windows and ROI capture the vertex N100-P200 complex, we observed a strong correlation between vertex N100 and P200 (Figure S7A,B; area under the curve (AUC) of LMFP; r(19)=0.91, $\mathrm{p}=0.00000004$; regression: $\mathrm{F}(1,19)=81.68, \mathrm{p}=0.00000004 ; \mathrm{R} 2=0.82)$.

To verify that sensory suppression techniques did not alter the early local TEP, we compared LMFP of the early window (14-86 ms) in electrodes local to the site of stimulation. For each condition, the AUC of the LMFP was utilized (and referred to simply as LMFP in the manuscript). Contrasts included No foam vs. Foam conditions (with other factors matched) and Jittered vs. Unjittered conditions (with other factors matched). To identify an ROI for examining local response to TMS, electrodes maximally different from baseline in the early window were chosen: AF3, AFz, F3, F1, FC3, FC1 (Section S1.2 and Figure S6C for ROI). We found no significant effect on the early TEP response (LMFP) of using Foam $(\mathrm{T}=-0.3534, \mathrm{DF}=19, \mathrm{p}=0.7277)$ or an Unjittered protocol $(\mathrm{T}=-0.5773$, $\mathrm{DF}=19, \mathrm{p}=0.5705 ;$ Figure $1 \mathrm{C}-\mathrm{F})$.

To ensure that an unjittered ISI did not induce changes in the early local TEP over time, we compared the first half of trials to the second half in the Unjittered condition [60,61]. We observed no significant difference in the early LMFP between the first half and second half of trials in the Unjittered (2 second ISI) condition (Figure S6; T=1.2542, DF=19, $\mathrm{p}=0.2250, \mathrm{CI}=-11.4509,45.6910)$.

\subsubsection{Statistical analyses of TEPs}


To compare single pulse TEP responses across the three masking protocols, we computed the LMFP for the N100 and P200 time windows in the central ROI. We performed an Analysis of Variance (ANOVA, repeated measures) with three levels (No masking, Standard masking, ATTENUATE), followed by post hoc pairwise comparisons using Tukey's HSD procedure where appropriate.

\subsection{Perceptual Ratings}

To assess perceptual experience during each stimulation condition, participants were asked to respond verbally immediately following each condition to rate loudness, scalp sensation, and pain perception on scales ranging from 0 to 10 . These scores were inputted into the research electronic data capture system (REDCAP, Vanderbilt, Nashville, TN). To ensure consistency in how these questions were phrased across conditions and subjects, the following scripts were used:

With 0 being you could not hear it, and 10 being as loud as a fire alarm, how loud did you perceive the 'click' sound to be?

With 0 being you could not feel it, and 10 being it felt as intense as a hard flick, how much did you feel the tapping sensation?

With 0 being no pain at all, and 10 being unbearable pain, how much pain did you feel?

\subsubsection{Statistical analyses of perceptual ratings}

Raw perceptual ratings were compared across the three conditions using a repeated measures ANOVA with three levels (No masking, Standard masking, ATTENUATE), followed by post hoc pairwise comparisons using Tukey's HSD procedure where appropriate.

2.5. Interactions between perceptual ratings and vertex N100-P200. To further understand the relationship between perceptual ratings of loudness, scalp sensation, pain, and the vertex N100-P200, an exploratory analysis compared perceptual ratings and vertex N100-P200 LMFP values across subjects for the No masking condition only. The goal of this analysis was to better understand the relationship between unsuppressed sensory contributions. For this analysis, a Pearson's correlation matrix was generated with correlation coefficients (Figure S7), and follow-up linear univariate regression analyses were performed for significantly correlated factors using the regress function in MATLAB R2021a (Mathworks, Natick, MA, USA) [62,63].

\section{Results}

3.1. The ATTENUATE protocol is superior to Standard masking at reducing the vertex N100-P200.

The vertex N100 and P200 LMFPs (see Section 2.3.2 above for ROI and window selection) were compared across the three sensory suppression protocols (No masking, Standard masking, and ATTENUATE). Vertex LMFP was significantly different across conditions 
in the $\mathrm{N} 100(\mathrm{~F}(2,57)=3.64, \mathrm{p}=0.03)$ and $\mathrm{P} 200(\mathrm{~F}(2,57)=9.40, \mathrm{p}=0.0003)$ time windows (Figure 2B-D). Post-hoc pairwise comparisons revealed that ATTENUATE reduced the LMFP vertex $\mathrm{N} 100(\mathrm{M}=145.84, \mathrm{SD}=62.13)$ compared to No masking $(\mathrm{M}=304.28$, $\mathrm{SD}=288.54$; Tukey's HSD, $\mathrm{p}=0.03$ ). Standard masking did not show statistical differences from No masking $(\mathrm{M}=236.38, \mathrm{SD}=167.59 ; \mathrm{p}=0.52)$ or from ATTENUATE $(\mathrm{p}=0.26)$. ATTENUATE reduced the LMFP vertex P200 (M=311.98, SD=130.51) compared to No masking $(\mathrm{M}=727.68, \mathrm{SD}=401.13 ; \mathrm{p}=0.0002)$ and compared to Standard masking $(\mathrm{M}=563.90, \mathrm{SD}=309.63 ; \mathrm{p}=0.03)$. Standard masking did not show statistical differences from No masking $(\mathrm{p}=0.21)$. These results reflect that ATTENUATE reduced vertex N100 by $54.41 \%$ and vertex P200 by $56.58 \%$ from No masking (average of $55.94 \%$ reduction across the vertex N100-P200 complex). In comparison, Standard masking reduced vertex $\mathrm{N} 100$ by $22.31 \%$ and vertex P200 by $22.51 \%$ (average of $22.45 \%$ across the vertex N100P200 complex). In summary, we observed a significant group effect across sensory suppression procedures and ATTENUATE reduced the vertex N100 and P200 more than Standard masking.

\subsection{ATTENUATE is more effective than Standard masking at reducing loudness perception and scalp sensation.}

Raw perceptual ratings for loudness of "click", sensation on the scalp, and pain were compared across the three masking protocols (No masking, Standard masking, and ATTENUATE). See Figure 2A for perceptual ratings following Standard masking and ATTENUATE conditions. We found a significant difference in loudness perception $(\mathrm{F}(2,57)=8.53, \mathrm{p}=0.0006)$ and scalp sensation $(\mathrm{F}(2,57)=5.47, \mathrm{p}=0.0067)$ across conditions (Figure 2A). Perception of pain did not change between the conditions $(F(2,57)=0.06, p$ $=0.9461)$. Post-hoc pairwise comparisons revealed that ATTENUATE reduced loudness rating $(\mathrm{M}=2.24, \mathrm{SD}=1.51 ; \mathrm{p}=0.0004)$ and scalp sensation $(\mathrm{M}=4.15, \mathrm{SD}=2.10 ; \mathrm{p}=0.006)$ compared to No masking (Loudness: $\mathrm{M}=4.23$, $\mathrm{SD}=1.92$; Scalp: $\mathrm{M}=6.30, \mathrm{SD}=2.36$ ). Standard masking (Loudness: $\mathrm{M}=3.08, \mathrm{SD}=1.51$; Scalp: $\mathrm{M}=4.78, \mathrm{SD}=2.07$ ) did not show a statistical difference from No masking (Loudness: $\mathrm{p}=0.07$; Scalp: $\mathrm{p}=0.08$ ) or from ATTENUATE (Loudness: $\mathrm{p}=0.15$; Scalp: $\mathrm{p}=0.56$ ). These results reflect that ATTENUATE reduced loudness rating by $50.30 \%$ and scalp sensation by $35.52 \%$ from No masking. In comparison, Standard masking reduced loudness rating by $27.22 \%$ and scalp sensation by $24.21 \%$. In summary, we observed a significant group effect across sensory suppression procedures with ATTENUATE reducing the perception of "click" loudness and scalp sensation compared with No masking.

\subsection{Individual auditory, foam, or ISI timing modifications are not sufficient for reducing vertex $\mathbf{N 1 0 0 - P 2 0 0}$ or sensory perception.}

To determine if components of these sensory suppression modifications in isolation reduce the vertex N100-P200 or sensory perception, we compared vertex N100-P200 LMFP and perceptual ratings across auditory (No noise, Noise, Noise and over-the-ear protection; Figure 3A-D), foam (No foam, Foam; Figure 3E-H), and ISI timing (Jittered, Unjittered; Figure 3I-L) conditions. For each comparison, all other modifications were matched.

\subsubsection{Auditory suppression}


An ANOVA across the three auditory conditions (No noise, Noise, Noise and over-the-ear protection) revealed no effect on vertex N100 (Figure 3C; F(2,57) $=1.27, \mathrm{p}=0.29$ ) with an insignificant but marginal effect on vertex P200 (Figure 3D; $F(2,57)=3.10, p=0.05$ ). Auditory suppression had an effect on loudness rating across the three conditions (Figure $3 \mathrm{E} ; \mathrm{F}(2,57)=6.12, \mathrm{p}=0.0039)$. Post-hoc pairwise comparisons revealed that Noise with over-the-ear protection reduced loudness $(\mathrm{M}=2.25, \mathrm{SD}=1.59)$ compared to No noise $(\mathrm{M}=4.23, \mathrm{SD}=1.92$; Tukey's HSD; $\mathrm{p}=0.003)$. Noise alone did not reduce loudness rating $(\mathrm{M}=3.10, \mathrm{SD}=1.84)$ from No noise $(\mathrm{p}=0.12)$ but was also not different from Noise with over-the-ear protection $(\mathrm{p}=0.30)$. Auditory suppression protocols did not reduce scalp sensation (Figure $3 \mathrm{~F} ; \mathrm{F}(2,57)=3.08, \mathrm{p}=0.05$ ) or pain rating (Figure $3 \mathrm{G} ; \mathrm{F}(2,57)=0.56, \mathrm{p}=$ $0.5730)$.

\subsubsection{Foam}

The use of Foam had no effect on vertex N100 (Figure 3J; T $=-0.2886, \mathrm{DF}=19, \mathrm{p}=0.7760$, $\mathrm{CI}=-46.9654,35.5838)$ or vertex $\mathrm{P} 200(\mathrm{Fig} 3 \mathrm{~K} ; \mathrm{T}=-0.8277, \mathrm{DF}=19, \mathrm{p}=0.4181, \mathrm{CI}=-$ 122.5488, 53.0926). Foam also had no effect on loudness rating (Figure $3 \mathrm{~L} ; \mathrm{T}=1.0918$, $\mathrm{DF}=19, \mathrm{p}=0.2886 ; \mathrm{CI}=-0.3210,1.0210$ ), scalp sensation (Figure $3 \mathrm{M} ; \mathrm{T}=1.4690, \mathrm{DF}=19$, $\mathrm{p}=0.1582 ; \mathrm{CI}=-0.3398,1.9398$ ), or pain rating (Figure $3 \mathrm{~N} ; \mathrm{T}=1.5305, \mathrm{DF}=19, \mathrm{p}=$ $0.1424 ; \mathrm{CI}=-0.2114,1.3614)$.

\subsubsection{ISI Timing}

Using an Unjittered ISI had a non-significant suppressive effect on vertex N100 (Figure $3 \mathrm{Q} ; \mathrm{T}=1.8574, \mathrm{DF}=19, \mathrm{p}=0.0788, \mathrm{CI}=-7.5349,126.3170)$ and a significant suppressive effect on vertex P200 (Figure 3R; $\mathrm{T}=3.8362$, $\mathrm{DF}=19, \mathrm{p}=0.0011, \mathrm{CI}=78.0444,265.4598$ ). Unjittered ISI had no effect on loudness rating (Figure 3S; T $=-0.8193, \mathrm{DF}=19, \mathrm{p}=0.4228$; $\mathrm{CI}=-0.8443,0.3693$ ), scalp sensation (Figure 3T; $\mathrm{T}=-1.1981, \mathrm{DF}=19, \mathrm{p}=0.2456 ; \mathrm{CI}=-$ $1.6482,0.4482$ ), or pain rating (Figure $3 \mathrm{~W} ; \mathrm{T}=-0.0901, \mathrm{DF}=19, \mathrm{p}=0.9291 ; \mathrm{CI}=-0.6056$, $0.5556)$.

In summary, auditory, foam, or ISI timing modifications alone are only minimally effective strategies for reducing vertex N100-P200 LMFP and perceptual ratings of "click" loudness, scalp sensation, or pain.

\subsection{Pain may be contributing to vertex N100-P200.}

Finally, to better understand the relationship between electrophysiology and perception, we compared the vertex N100, vertex P200, and perceptual ratings in the No masking condition (Figure S7) using a correlation matrix of all measures. All relationships were insignificant except for between N100 and P200 as well as between Pain and the P200 (Figure S7A; Methods section 2.3.2). Pain rating had a positive correlation with vertex P200 (Figure S7C; correlation: $r(19)=0.45$, $p=0.04$; regression: $F(1,19)=4.67, p=0.04 ; R^{2}$ $=0.20)$.

\section{Discussion}


In the present study, to reduce the sensory effects of TMS, we sought to experimentally minimize the vertex N100-P200 and sensory perception arising from suprathreshold TMS to the dlFPC. We developed a novel combination of experimental sensory suppression techniques, termed ATTENUATE, which consisted of auditory noise masking, foam, overthe-ear protection, and unjittered pulse timing. To our knowledge, this is the first study to present the ATTENUATE protocol. We find the following: 1) The ATTENUATE protocol significantly reduced the vertex N100-P200 by 56\%, outperforming other standard masking procedures, with no effect on the early TEP; 2) The ATTENUATE protocol reduced "click" loudness rating by $50 \%$ and scalp sensation by $36 \%$, outperforming standard approaches; and 3) Single sensory experimental modifications alone are not sufficient to significantly reduce vertex N100-P200 or sensory perception.

We show that additional experimental modifications above noise masking alone are needed to reduce the N100-P200 after supratheshold TMS to dlPFC (Figure 3A-G). Compared to prior studies that suggest that noise masking alone can minimize the sensory TEP $[2,19]$, our study differs by intensity and brain target. Regarding intensity, compared to previous work that focused on subthreshold intensities (90\% rMT: [2,19]), our suprathreshold stimulation protocol (120\% rMT) better mimicked clinical stimulation parameters [64], but is more difficult to mask $[18,40,41,65]$. In regards to brain target, while other studies have explored sensory suppression after TMS to the primary motor [19] or premotor [2] targets, here we focus on the dIPFC, which may have different sensory contributions to the vertex N100-P200 compared with motor targets $[43,66]$.

While these differences in intensity ( 80 vs. $120 \%$ rMT) or brain target (premotor/M1 vs. dlPFC) may partially explain the inability to fully suppress the vertex N100-P200, other factors may be contributing. The link between sensory potentials, brain target, and intensity is not yet clear [43]. TEPs from M1 stimulation highly correlate with those from non-brain regions (shoulder), regardless of stimulation intensity (120\% vs 80\% rMT) [18], suggesting that peripherally evoked contributions to the TEP may be considerable regardless of stimulation intensity or target. Multiple studies have demonstrated that N100-P200 components can persist, both for subthreshold and suprathreshold M1 stimulation, even after suppression of TMS click perception $[65,67]$. Therefore, we cannot conclude that auditory suppression protocols will be effective for all designs, nor that auditory perception of the "click" will be an effective indication of suppression of sensory components in the TEP. We may be yet to find the most effective sensory suppression protocol for all designs and populations. However, our proposed novel combination of sensory reduction procedures (ATTENUATE), which includes extra auditory reduction (with the use of overthe-ear protection), foam, and predictable timing of TMS pulses, is superior at reducing vertex N100-P200 and sensory perception (loudness and scalp feeling) compared to standard experimental procedures.

\subsection{Effect of a foam spacer on the sensory TEP}

We find that a foam spacer attached to the bottom of the coil had no effect on vertex N100P200, "click" loudness perception, scalp feeling or pain. Although foam could be contributing to the combined effectiveness of ATTENUATE, there was no impact when 
other modifications were matched (Figure 3H-N). Foam has been suggested to reduce bone conduction of the TMS "click" sound [23] and shown to be effective when used in combination with auditory suppression methods [41]. As such, foam padding between the coil and scalp has become standard procedure to help suppress the vertex N100-P200. However, foam also increases coil to cortex distance and there are no guidelines for adjusting stimulation intensity to account for this increased distance or for reporting whether foam was used in determining motor thresholds. Coil to cortex distance has a strong influence on induced electric field in cortex [68] - enough to significantly increase MT determination [41], as also observed in the current study (Table S1). The lack of reduction in vertex N100-P200 with foam in our conditions when other factors were matched could be due to this increased intensity of TMS with compared to without foam. Interestingly, we also observed no difference in early localized TEP, suggesting that the adjusted rMT with foam likely resulted in a matched induced electrical field, potentially diminishing the argument that the adjusted rMT accounts for our lack of suppression. Overall, our results suggest that foam may not be alone effective for reducing sensory confounds. If used it is important that stimulation intensity is adjusted to account for the increased distance from coil to cortex. Furthermore, this adjusted rMT should be reported in future studies to allow further analysis into this critical question.

\subsection{Non-modal or multimodal component contributions to the TEP}

The vertex N100-P200 complex has been described as an auditory component (see AEP; $[23,44])$, but it is likely to have multimodal sensory contributions. Although observed in the TEP, vertex N100-P200 complexes with similar/matching time course of peak latencies and similar source activations have been more rigorously examined and described in response to sensory stimuli other than the TMS "click" sound. Many of these studies describe multisensory or cross-modal impacts on the vertex N100-P200 [69-71], suggesting that it is not modality specific and instead largely determined by the intrinsic saliency of the stimulus and its task relevance [28,72].

In TMS-EEG experiments, it is difficult to distinguish between unimodal auditory and nonmodal or multimodal sensory contributions to the vertex N100-P200. In addition, it is unclear if sensory contributions are likely to sum linearly. In light of this, sensory suppression protocols for TMS-EEG may be more effective if the vertex N100-P200 is assumed to be multimodal. Our proposed ATTENUATE procedure may demonstrate additional benefit over a Standard masking procedure due to over-the-ear auditory masking or saliency reduction through predictable ISI of the TMS pulses. Of note is that neither auditory masking (even with over-the-ear protection) nor predictable ISI timing was more than borderline or minimally effective at suppressing vertex N100-P200 when used alone. Instead, maximal suppression was achieved when combining auditory masking and predictable ISI timing, suggesting that a combined sensory masking and sensory attenuation protocol is most effective.

4.3. Is sensory suppression the most effective strategy for reducing vertex N100-P200? One clear limitation of our results is that neither perceptual ratings nor vertex N100-P200 were fully eliminated. It should be noted that our design was intended to evoke a large 
vertex N100-P200 by using suprathreshold stimulation (120\% rMT) and with a stimulation target that is known to induce significant sensory artifact [66]. Future work should examine the efficacy of the ATTENUATE protocol across stimulation intensities and targets. ATTENUATE may fully eliminate vertex N100-P200 at lower stimulation intensities or other stimulation targets, but this is outside the scope of the current work and will need to be investigated experimentally.

Furthermore, when the study design allows, sensory suppression techniques should be considered only after other experimental options such as active controls. For instance, if the experimental question allows for conditions with matched intensity, matched sensory suppressive protocols, and target locations with active TMS, then perception and cortical sensory components in the TEP should also be matched. Although this design is optimal, it is not feasible for many studies, either due to time or other constraints. Alternatively, one can isolate the sensory contributions to the TEP using sensory-matched sham protocols. Although it is difficult to match the sensory experience of active TMS with sham TMS, the topography and time course of evoked sensory potentials may be similar [18] enough to employ an ICA-based technique for removal [40,73]. Indeed, a combination of sensory suppression, such as ATTENUATE, coupled with sensory-matched sham TMS may be most effective for reducing the impact of sensory confounds while ensuring that residual sensory contributions to TEP can be more easily identified.

\subsection{Sensory potentials and pain perception}

Our results suggest that perception of pain due to TMS may be relevant to the vertex N100P200 complex. This result is perhaps unsurprising as previous work has demonstrated substantial overlap between auditory/somatosensory responses and activity in a 'pain matrix' network with nociceptive stimuli applied to the skin [28]. Due to a high correlation between the response to sensory and nociceptive stimulation as well as sensory/nociceptive responses and saliency ratings, the authors suggested that sensory responses and pain matrix activity may be best characterized as stimulus saliency-related network activity. Although our correlation and regression analyses were exploratory, this work suggests that reducing the saliency of TMS, including minimization of pain, should be investigated to minimize the vertex N100-P200.

\section{Future directions}

While this work provides critical improvements in sensory suppression during TMS studies, several important questions remain. Given the wide variety of acoustic and somatosensory responses to TMS, the ATTENUATE protocol should be tested with a range of stimulation intensities, coils, and brain targets to establish its efficacy for different stimulation environments. It is also important to explore how ATTENUATE performs in patients with sensory deficits such as hearing loss and sensory processing disorders. Additionally, our data suggest that predictability of TMS pulse timing can contribute to amplitude suppression in sensory TEP, building on prior work showing MEP attenuation with predictable M1 stimulation [45,46]. Although we did not observe a cumulative effect on the TEP using 80 unjittered single pulses of TMS, the sensory predictive suppressive effect should be examined with more single pulses and with a range of unjittered ISIs to 
ensure that the unjittered protocol does not induce a buildup of brain changes (i.e. neuroplasticity). Sensorimotor prediction for the timing of sensory events is well documented [See [74] and [75] for reviews], and may account for motor and sensory attenuation with predictable TMS pulse timing. However, the suggestion that the principles of sensorimotor timing can be used to optimize non-motor and non-sensory TEP is novel to the best of our knowledge. Future work should compare the effects on the TEP of taskrelevant sensorimotor experience [46,83], readiness-to-act [84,85], and interval and phase timing in rhythmically predictable TMS sequences [74,76-82], as variables in the TMS sensory predictive suppressive effect.

\section{Conclusions}

We investigated the electrophysiological and perceptual consequences of applying different sensory suppression protocols with suprathreshold TMS to dlPFC. We find that ATTENUATE outperforms the Standard masking protocol for reducing both the vertex N100-P200 and sensory perception. Further, our data support that auditory suppression, foam spacing, or pulse timing alone are not sufficient to reduce the vertex N100-P200, likely due to the non-modal or multimodal contributions of the sensory experience of the TMS pulse.

\section{Acknowledgements.}

We extend gratitude to all of our research participants. We would also like to acknowledge the generous contributions of the members of the Personalized Neurotherapeutics Laboratory for helpful feedback on the manuscript and throughout the course of the study.

This research was supported by the National Institute of Mental Health under award number R01MH126639 and a Burroughs Wellcome Fund Career Award for Medical Scientists (CJK).

JMR was supported by the Department of Veterans Affairs Office of Academic Affiliations Advanced Fellowship Program in Mental Illness Research and Treatment, the Medical Research Service of the Veterans Affairs Palo Alto Health Care System and the Department of Veterans Affairs Sierra-Pacific Data Science Fellowship.

\section{Declaration of Interest.}

CJK holds equity in Alto Neuroscience, Inc.

\section{Appendix A}

Supplementary data related to this article can be found online.

\section{Data availability.}

Data will be available on NIMH Data Archive within 6 months of publication. 

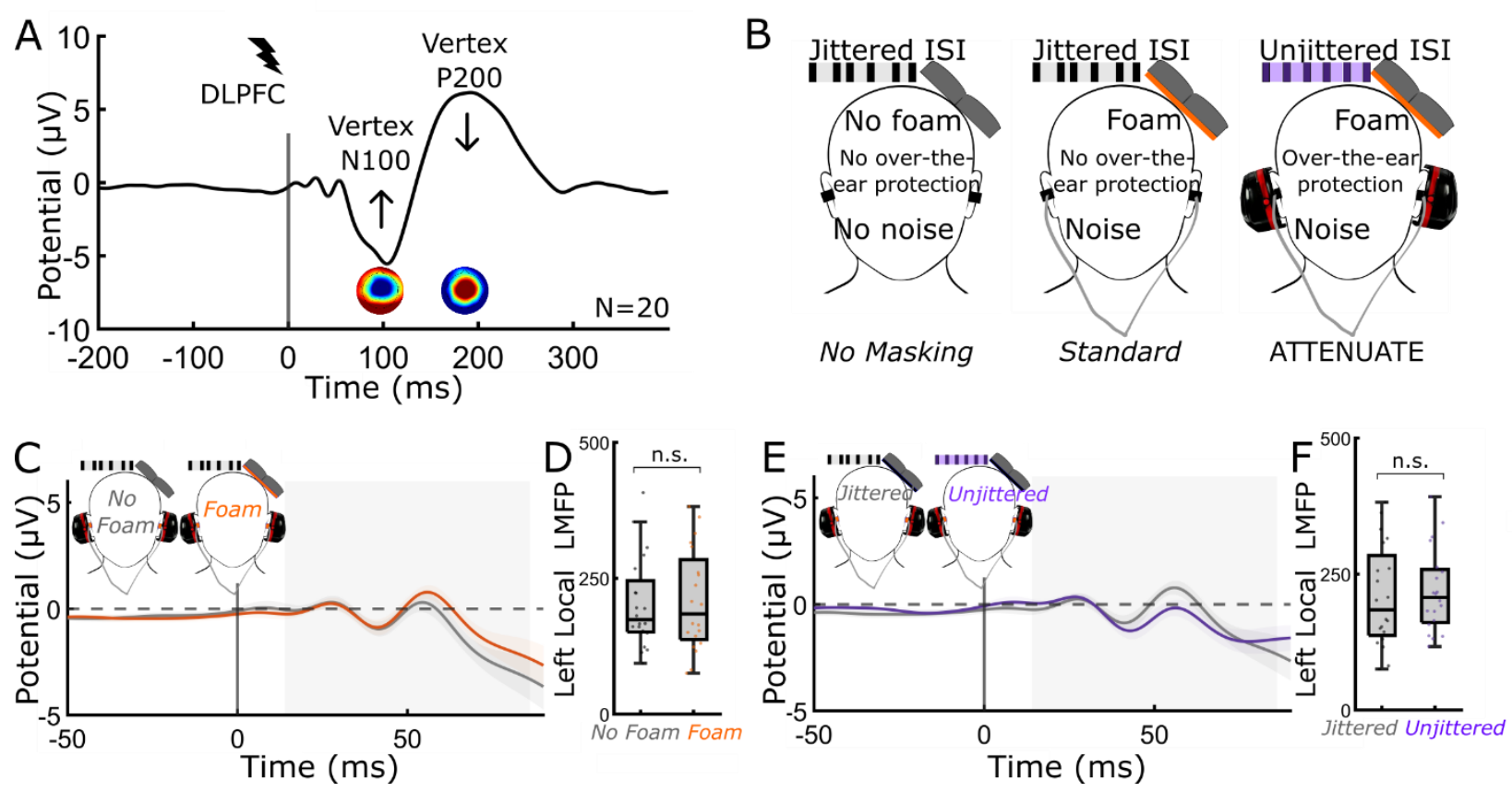

Figure 1. Experimental design. (A) Single pulse TMS-EEG to the left dlPFC. TMS was applied at $120 \%$ rMT (80 trials). Perceptual reports of loudness of the TMS "click", intensity of scalp sensation, and pain followed each condition. Vertex N100-P200 was quantified using LMFP. Arrows denote that the goal of the study was to minimize the N100-P200 and sensory perception while preserving the early TEP. (B) Three experimental conditions were compared: No masking (jittered ISI/no foam/no noise/no over-the-ear protection), Standard masking (jittered ISI/foam/noise/no over-the-ear protection), and ATTENUATE (unjittered ISI/foam/noise/over-the-ear protection). (C-F) Neither foam (CD) nor unjittered ISI (E-F) altered the early local TEP (14-86 ms). (C-D) Effect of foam on early (14-86ms) local TEP. Foam did not modify the early local TEP $(\mathrm{T}=-0.35$, DF $=19$, $\mathrm{p}=0.73$ ). (E-F) Effect of using an unjittered ISI on early local TEP. Modifying the timing of TMS pulses did not change the early local TEP $(\mathrm{T}=-0.58, \mathrm{DF}=19, \mathrm{p}=0.57)$. All error bars denote standard error. N.S. = not significant. 

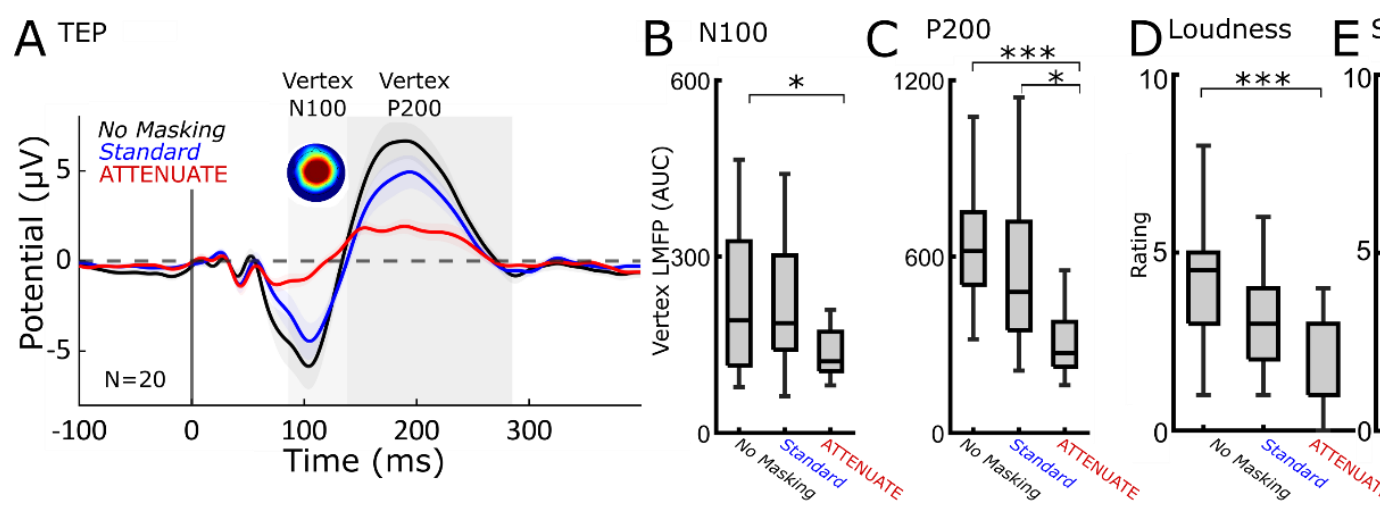

Figure 2. The ATTENUATE protocol is superior to Standard masking at reducing vertex N100-P200, “click" loudness perception, and scalp sensation. (A) Group mean TEPs of vertex ROI $(\mathrm{N}=20)$. Shaded areas indicate time windows used for analysis. $(\mathrm{B}-\mathrm{C})$ ATTENUATE reduces the vertex N100-P200. Vertex LMFP is reduced in both N100 $(\mathrm{F}(2,57)=3.64, \mathrm{p}=0.03)$ and $\mathrm{P} 200(\mathrm{~F}(2,57)=9.40, \mathrm{p}=0.0003)$ time windows across the three conditions. Pairwise comparisons revealed that ATTENUATE reduced vertex N100-P200 compared with No Masking (N100: p=0.03; P200: p=0.0002), and that ATTENUATE reduced vertex P200 compared with Standard masking ( $\mathrm{p}=0.03)$. Comparisons between No Masking and Standard masking were non-significant (N100 p=0.52; P200 p=0.21). (D-E) Sensory suppression protocols reduced perception of loudness $(F(2,57)=8.53, p=0.0006)$ and scalp sensation $(\mathrm{F}(2,57)=5.47, \mathrm{p}=0.007)$. Pairwise comparisons demonstrate that ATTENUATE reduced both loudness (loudness $\mathrm{p}=0.0004$ ) and scalp sensation (scalp sensation $\mathrm{p}=0.006$ ) from No masking, but did not reduce compared with Standard masking in loudness rating $(\mathrm{p}=0.15)$ or scalp sensation $(\mathrm{p}=0.56)$. Comparisons between No masking and Standard masking were non-significant (loudness $\mathrm{p}=0.07$, scalp sensation $\mathrm{p}=0.08$ ). $(\mathrm{F})$ Pain was not effected by protocol $(\mathrm{F}(2,57)=0.06, \mathrm{p}=0.9461)$. All significant pairwise comparisons are indicated with brackets and asterisks mark level of significance. ${ }^{*} \mathrm{p}<.05$, $* * \mathrm{p}<.01, * * * \mathrm{p}<.001$. All error bars denote standard error. 


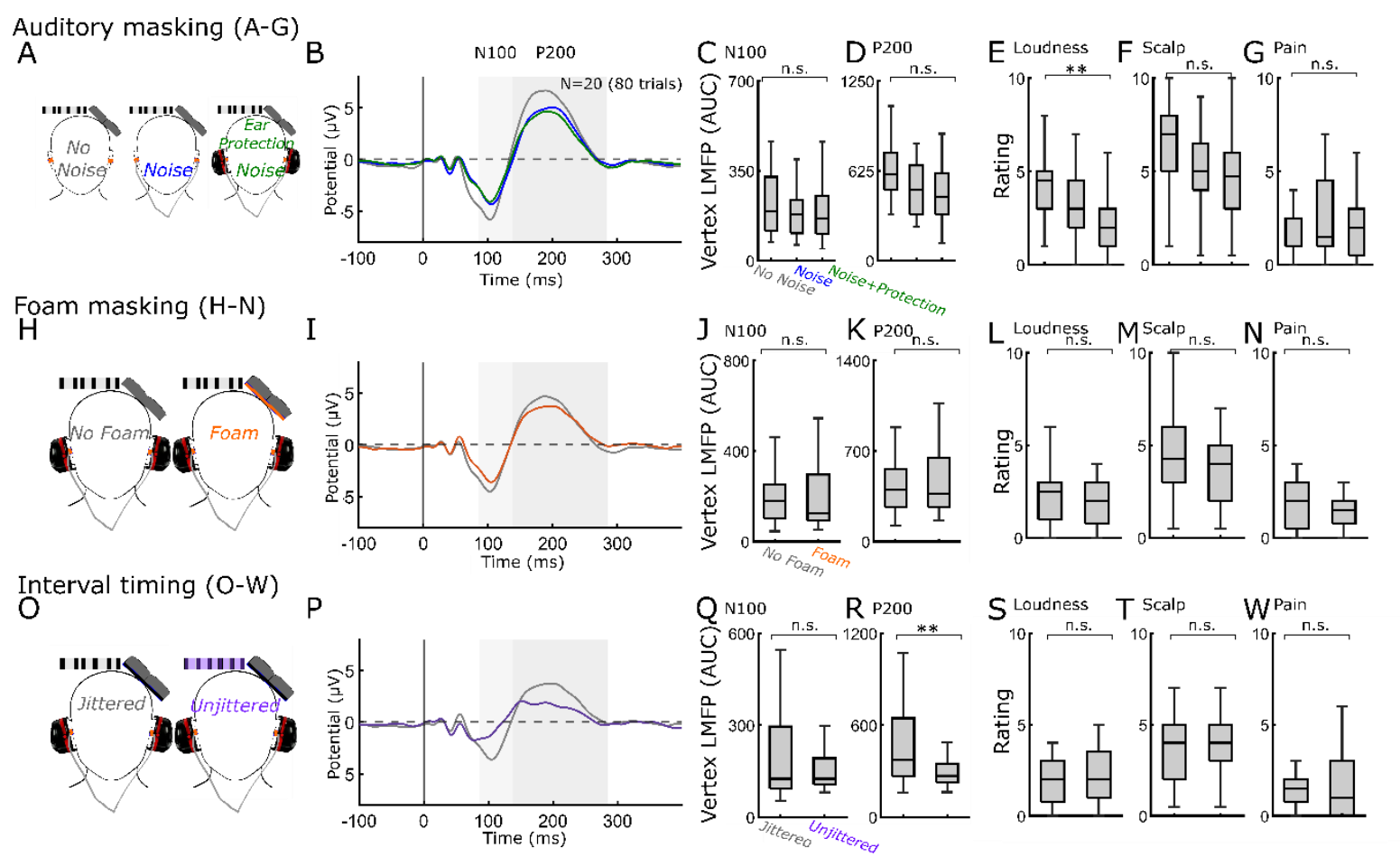

Figure 3. Individual auditory, foam, or timing modifications are only minimally effective for reducing vertex N100-P200 or sensory perception. (A) Auditory masking conditions: No noise, Noise, Noise with over-the-ear protection (B) TEPs from the vertex ROI (C-D) LMFP for N100 (C) and P200 (D) time windows. ANOVAs revealed that auditory masking protocols only had a marginal but insignificant effect on vertex P200. (E-G) Perceptual ratings of "click" loudness (E), scalp sensation (F), and pain (G). ANOVAs revealed that auditory masking protocols had an effect on loudness rating, driven by Noise with over-the-ear protection change from No masking, an insignificant reduction in scalp sensation, and no effect on pain. (H) No foam and Foam conditions (I) TEPs from the vertex ROI (J-K) LMFP. T-tests revealed that Foam had no effect on vertex N100 or P200. (L-N) Perceptual ratings of loudness (L), scalp sensation (M), and pain (N). T-tests revealed that Foam had no effect on any perceptual ratings. (O) Jittered and Unjittered ISI conditions (P) TEPs from the vertex ROI (Q-R) LMFP. T-tests revealed that using an Unjittered ISI had a non-significant effect in vertex N100, and a significant effect on vertex P200. (S-W) Perceptual ratings of loudness (S), scalp sensation (T), and pain (W). T-tests revealed that using an Unjittered ISI had no effect on any perceptual ratings. $\mathrm{p}<.05$, ** $\mathrm{p}<.01$, *** $\mathrm{p}<.001$. All error bars denote standard error. Shaded areas indicate time windows used for analysis. 


\section{References}

[1] Barker AT, Jalinous R, Freeston IL. NON-INVASIVE MAGNETIC STIMULATION OF HUMAN MOTOR CORTEX. The Lancet 1985;325:1106-7.

https://doi.org/10.1016/S0140-6736(85)92413-4.

[2] Massimini M, Ferrarelli F, Huber R, Esser SK, Singh H, Tononi G. Breakdown of Cortical Effective Connectivity During Sleep. Science 2005;309:2228-32. https://doi.org/10.1126/science.1117256.

[3] Ferrarelli F, Massimini M, Sarasso S, Casali A, Riedner BA, Angelini G, et al. Breakdown in cortical effective connectivity during midazolam-induced loss of consciousness. Proceedings of the National Academy of Sciences 2010;107:2681-6. https://doi.org/10.1073/pnas.0913008107.

[4] Fischer AS, Keller CJ, Etkin A. The Clinical Applicability of Functional Connectivity in Depression: Pathways Toward More Targeted Intervention. Biological Psychiatry: Cognitive Neuroscience and Neuroimaging 2016;1:262-70. https://doi.org/10.1016/j.bpsc.2016.02.004.

[5] Casarotto S, Määttä S, Herukka S-K, Pigorini A, Napolitani M, Gosseries O, et al. Transcranial magnetic stimulation-evoked EEG/cortical potentials in physiological and pathological aging. NeuroReport 2011;22:592-7. https://doi.org/10.1097/WNR.0b013e328349433a.

[6] Pascual-Leone A, Freitas C, Oberman L, Horvath JC, Halko M, Eldaief M, et al. Characterizing Brain Cortical Plasticity and Network Dynamics Across the Age-Span in Health and Disease with TMS-EEG and TMS-fMRI. Brain Topogr 2011;24:302-15. https://doi.org/10.1007/s10548-011-0196-8.

[7] Shafi MM, Vernet M, Klooster D, Chu CJ, Boric K, Barnard ME, et al. Physiological consequences of abnormal connectivity in a developmental epilepsy: Cortical Connectivity. Ann Neurol 2015;77:487-503. https://doi.org/10.1002/ana.24343.

[8] Rogasch NC, Daskalakis ZJ, Fitzgerald PB. Cortical Inhibition, Excitation, and Connectivity in Schizophrenia: A Review of Insights From Transcranial Magnetic Stimulation. Schizophrenia Bulletin 2014;40:685-96. https://doi.org/10.1093/schbul/sbt078.

[9] Kerwin LJ, Keller CJ, Wu W, Narayan M, Etkin A. Test-retest reliability of transcranial magnetic stimulation EEG evoked potentials. Brain Stimulation 2018;11:536-44. https://doi.org/10.1016/j.brs.2017.12.010.

[10] Ilmoniemi RJ, Virtanen J, Ruohonen J, Karhu J, Aronen HJ, Näätänen R, et al. Neuronal responses to magnetic stimulation reveal cortical reactivity and connectivity: NeuroReport 1997;8:3537-40. https://doi.org/10.1097/00001756199711100-00024.

[11] Rosanova M, Casali A, Bellina V, Resta F, Mariotti M, Massimini M. Natural Frequencies of Human Corticothalamic Circuits. Journal of Neuroscience 2009;29:7679-85. https://doi.org/10.1523/JNEUROSCI.0445-09.2009.

[12] Bortoletto M, Veniero D, Thut G, Miniussi C. The contribution of TMS-EEG coregistration in the exploration of the human cortical connectome. Neuroscience \& 
Biobehavioral Reviews 2015;49:114-24.

https://doi.org/10.1016/j.neubiorev.2014.12.014.

[13] Wu W, Keller CJ, Rogasch NC, Longwell P, Shpigel E, Rolle CE, et al. ARTIST: A fully automated artifact rejection algorithm for single-pulse TMS-EEG data. Hum Brain Mapp 2018;39:1607-25. https://doi.org/10.1002/hbm.23938.

[14] Eshel N, Keller CJ, Wu W, Jiang J, Mills-Finnerty C, Huemer J, et al. Global connectivity and local excitability changes underlie antidepressant effects of repetitive transcranial magnetic stimulation. Neuropsychopharmacol 2020;45:1018-25. https://doi.org/10.1038/s41386-020-0633-z.

[15] Rogasch NC, Fitzgerald PB. Assessing cortical network properties using TMSEEG. Hum Brain Mapp 2013;34:1652-69. https://doi.org/10.1002/hbm.22016. [16] Ozdemir RA, Tadayon E, Boucher P, Sun H, Momi D, Ganglberger W, et al. Cortical responses to noninvasive perturbations enable individual brain fingerprinting. Brain Stimulation 2021;14:391-403. https://doi.org/10.1016/j.brs.2021.02.005.

[17] Conde V, Tomasevic L, Akopian I, Stanek K, Saturnino GB, Thielscher A, et al. The non-transcranial TMS-evoked potential is an inherent source of ambiguity in TMS-EEG studies. NeuroImage 2019;185:300-12.

https://doi.org/10.1016/j.neuroimage.2018.10.052.

[18] Biabani M, Fornito A, Mutanen TP, Morrow J, Rogasch NC. Characterizing and minimizing the contribution of sensory inputs to TMS-evoked potentials. Brain Stimulation 2019;12:1537-52. https://doi.org/10.1016/j.brs.2019.07.009.

[19] Rocchi L, Di Santo A, Brown K, Ibáñez J, Casula E, Rawji V, et al. Disentangling EEG responses to TMS due to cortical and peripheral activations. Brain Stimulation 2021;14:4-18. https://doi.org/10.1016/j.brs.2020.10.011.

[20] Freedberg M, Reeves JA, Hussain SJ, Zaghloul KA, Wassermann EM. Identifying site- and stimulation-specific TMS-evoked EEG potentials using a quantitative cosine similarity metric. PLoS ONE 2020;15:e0216185. https://doi.org/10.1371/journal.pone.0216185.

[21] Siebner HR, Conde V, Tomasevic L, Thielscher A, Bergmann TO. Distilling the essence of TMS-evoked EEG potentials (TEPs): A call for securing mechanistic specificity and experimental rigor. Brain Stimulation 2019;12:1051-4. https://doi.org/10.1016/j.brs.2019.03.076.

[22] Belardinelli P, Biabani M, Blumberger DM, Bortoletto M, Casarotto S, David O, et al. Reproducibility in TMS-EEG studies: A call for data sharing, standard procedures and effective experimental control. Brain Stimulation 2019;12:787-90. https://doi.org/10.1016/j.brs.2019.01.010.

[23] Nikouline V, Ruohonen J, Ilmoniemi RJ. The role of the coil click in TMS assessed with simultaneous EEG. Clinical Neurophysiology 1999;110:1325-8. https://doi.org/10.1016/S1388-2457(99)00070-X.

[24] Lioumis P, Kičić D, Savolainen P, Mäkelä JP, Kähkönen S. Reproducibility of TMS-Evoked EEG responses. Hum Brain Mapp 2009;30:1387-96.

https://doi.org/10.1002/hbm.20608. 
[25] Gordon PC, Desideri D, Belardinelli P, Zrenner C, Ziemann U. Comparison of cortical EEG responses to realistic sham versus real TMS of human motor cortex. Brain Stimulation 2018;11:1322-30. https://doi.org/10.1016/j.brs.2018.08.003. [26] Gosseries O, Sarasso S, Casarotto S, Boly M, Schnakers C, Napolitani M, et al. On the Cerebral Origin of EEG Responses to TMS: Insights From Severe Cortical Lesions. Brain Stimulation 2015;8:142-9. https://doi.org/10.1016/j.brs.2014.10.008.

[27] Harquel S, Bacle T, Beynel L, Marendaz C, Chauvin A, David O. Mapping dynamical properties of cortical microcircuits using robotized TMS and EEG: Towards functional cytoarchitectonics. NeuroImage 2016;135:115-24. https://doi.org/10.1016/j.neuroimage.2016.05.009.

[28] Mouraux A, Diukova A, Lee MC, Wise RG, Iannetti GD. A multisensory investigation of the functional significance of the "pain matrix." NeuroImage 2011;54:2237-49. https://doi.org/10.1016/j.neuroimage.2010.09.084.

[29] Čeponien $\doteq \mathrm{R}$, Rinne T, Näätänen R. Maturation of cortical sound processing as indexed by event-related potentials. Clinical Neurophysiology 2002;113:870-82. https://doi.org/10.1016/S1388-2457(02)00078-0.

[30] Sharma A, Kraus N, J. McGee T, Nicol TG. Developmental changes in P1 and N1 central auditory responses elicited by consonant-vowel syllables.

Electroencephalography and Clinical Neurophysiology/Evoked Potentials Section 1997;104:540-5. https://doi.org/10.1016/S0168-5597(97)00050-6.

[31] Eggermont JJ, Ponton CW, Don M, Waring MD, Kwong B. Maturational Delays in Cortical Evoked Potentials in Cochlear Implant Users. Acta Oto-Laryngologica 1997;117:161-3. https://doi.org/10.3109/00016489709117760.

[32] Knight RT, Hillyard SA, Woods DL, Neville HJ. The effects of frontal and temporal-parietal lesions on the auditory evoked potential in man.

Electroencephalography and Clinical Neurophysiology 1980;50:112-24. https://doi.org/10.1016/0013-4694(80)90328-4.

[33] Näätänen R, Picton T. The N1 Wave of the Human Electric and Magnetic Response to Sound: A Review and an Analysis of the Component Structure. Psychophysiology 1987;24:375-425. https://doi.org/10.1111/j.14698986.1987.tb00311.x.

[34] Siddiqi SH, Schaper FLWVJ, Horn A, Hsu J, Padmanabhan JL, Brodtmann A, et al. Brain stimulation and brain lesions converge on common causal circuits in neuropsychiatric disease. Nat Hum Behav 2021;5:1707-16.

https://doi.org/10.1038/s41562-021-01161-1.

[35] O'Reardon JP, Solvason HB, Janicak PG, Sampson S, Isenberg KE, Nahas Z, et al. Efficacy and safety of transcranial magnetic stimulation in the acute treatment of major depression: a multisite randomized controlled trial. Biol Psychiatry 2007;62:1208-16. https://doi.org/10.1016/j.biopsych.2007.01.018.

[36] Padberg F, George MS. Repetitive transcranial magnetic stimulation of the prefrontal cortex in depression. Exp Neurol 2009;219:2-13.

https://doi.org/10.1016/j.expneurol.2009.04.020.

[37] George MS, Wassermann EM, Williams WA, Callahan A, Ketter TA, Basser P, et al. Daily repetitive transcranial magnetic stimulation (rTMS) improves mood in 
depression. Neuroreport 1995;6:1853-6. https://doi.org/10.1097/00001756$199510020-00008$.

[38] Pascual-Leone A, Rubio B, Pallardó F, Catalá MD. Rapid-rate transcranial magnetic stimulation of left dorsolateral prefrontal cortex in drug-resistant depression. Lancet 1996;348:233-7. https://doi.org/10.1016/s01406736(96)01219-6.

[39] Fox MD, Buckner RL, White MP, Greicius MD, Pascual-Leone A. Efficacy of Transcranial Magnetic Stimulation Targets for Depression Is Related to Intrinsic Functional Connectivity with the Subgenual Cingulate. Biological Psychiatry 2012;72:595-603. https://doi.org/10.1016/j.biopsych.2012.04.028.

[40] Ross JM, Ozdemir RA, Lian SJ, Fried PJ, Schmitt EM, Inouye SK, et al. A structured ICA-based process for removing auditory evoked potentials. Scientific Reports 2021.

[41] ter Braack EM, de Vos CC, van Putten MJAM. Masking the Auditory Evoked Potential in TMS-EEG: A Comparison of Various Methods. Brain Topogr 2015;28:520-8. https://doi.org/10.1007/s10548-013-0312-z.

[42] Tchumatchenko T, Reichenbach T. A cochlear-bone wave can yield a hearing sensation as well as otoacoustic emission. Nat Commun 2014;5:4160.

https://doi.org/10.1038/ncomms5160.

[43] Herring JD, Thut G, Jensen O, Bergmann TO. Attention Modulates TMS-Locked Alpha Oscillations in the Visual Cortex. Journal of Neuroscience 2015;35:14435-47. https://doi.org/10.1523/JNEUROSCI.1833-15.2015.

[44] Ilmoniemi RJ, Kičić D. Methodology for Combined TMS and EEG. Brain Topogr 2010;22:233-48. https://doi.org/10.1007/s10548-009-0123-4.

[45] Tran DMD, McNair NA, Harris JA, Livesey EJ. Expected TMS excites the motor system less effectively than unexpected stimulation. NeuroImage 2021;226:117541. https://doi.org/10.1016/j.neuroimage.2020.117541.

[46] Stupacher J, Hove MJ, Novembre G, Schütz-Bosbach S, Keller PE. Musical groove modulates motor cortex excitability: A TMS investigation. Brain and Cognition 2013;82:127-36. https://doi.org/10.1016/j.bandc.2013.03.003. [47] Stokes MG, Chambers CD, Gould IC, Henderson TR, Janko NE, Allen NB, et al. Simple Metric For Scaling Motor Threshold Based on Scalp-Cortex Distance: Application to Studies Using Transcranial Magnetic Stimulation. Journal of Neurophysiology 2005;94:4520-7. https://doi.org/10.1152/jn.00067.2005. [48] Pridmore S, Fernandes Filho JA, Nahas Z, Liberatos C, George MS. Motor threshold in transcranial magnetic stimulation: a comparison of a neurophysiological method and a visualization of movement method. J ECT 1998;14:25-7.

[49] Chen AC, Oathes DJ, Chang C, Bradley T, Zhou Z-W, Williams LM, et al. Causal interactions between fronto-parietal central executive and default-mode networks in humans. Proceedings of the National Academy of Sciences 2013;110:19944-9. https://doi.org/10.1073/pnas.1311772110. [50] Janssen AM, Oostendorp TF, Stegeman DF. The coil orientation dependency of the electric field induced by TMS for M1 and other brain areas. J 
NeuroEngineering Rehabil 2015;12:47. https://doi.org/10.1186/s12984-015-00362.

[51] Laakso I, Hirata A, Ugawa Y. Effects of coil orientation on the electric field induced by TMS over the hand motor area. Phys Med Biol 2014;59:203-18. https://doi.org/10.1088/0031-9155/59/1/203.

[52] Tervo AE, Nieminen JO, Lioumis P, Metsomaa J, Souza VH, Sinisalo H, et al. Closed-loop optimization of transcranial magnetic stimulation with electroencephalography feedback. Bioengineering; 2021. https://doi.org/10.1101/2021.08.31.458148.

[53] Brasil-Neto JP, Cohen LG, Panizza M, Nilsson J, Roth BJ, Hallett M. Optimal focal transcranial magnetic activation of the human motor cortex: effects of coil orientation, shape of the induced current pulse, and stimulus intensity. J Clin Neurophysiol 1992;9:132-6.

[54] Mills KR, Boniface SJ, Schubert M. Magnetic brain stimulation with a double coil: the importance of coil orientation. Electroencephalography and Clinical Neurophysiology/Evoked Potentials Section 1992;85:17-21. https://doi.org/10.1016/0168-5597(92)90096-T.

[55] Siebner HR, Bergmann TO, Bestmann S, Massimini M, Johansen-Berg H, Mochizuki $\mathrm{H}$, et al. Consensus paper: Combining transcranial stimulation with neuroimaging. Brain Stimulation 2009;2:58-80. https://doi.org/10.1016/j.brs.2008.11.002.

[56] Delorme A, Makeig S. EEGLAB: an open source toolbox for analysis of singletrial EEG dynamics including independent component analysis. Journal of Neuroscience Methods 2004;134:9-21. https://doi.org/10.1016/j.jneumeth.2003.10.009.

[57] Bertazzoli G, Esposito R, Mutanen TP, Ferrari C, Ilmoniemi RJ, Miniussi C, et al. The impact of artifact removal approaches on TMS-EEG signal. NeuroImage 2021;239:118272. https://doi.org/10.1016/j.neuroimage.2021.118272.

[58] Atluri S, Frehlich M, Mei Y, Garcia Dominguez L, Rogasch NC, Wong W, et al. TMSEEG: A MATLAB-Based Graphical User Interface for Processing Electrophysiological Signals during Transcranial Magnetic Stimulation. Front Neural Circuits 2016;10. https://doi.org/10.3389/fncir.2016.00078.

[59] Rogasch NC, Sullivan C, Thomson RH, Rose NS, Bailey NW, Fitzgerald PB, et al. Analysing concurrent transcranial magnetic stimulation and electroencephalographic data: A review and introduction to the open-source TESA software. NeuroImage 2017;147:934-51. https://doi.org/10.1016/j.neuroimage.2016.10.031.

[60] Huang Y, Hajnal B, Entz L, Fabó D, Herrero JL, Mehta AD, et al. Intracortical Dynamics Underlying Repetitive Stimulation Predicts Changes in Network Connectivity. J Neurosci 2019;39:6122-35. https://doi.org/10.1523/JNEUROSCI.0535-19.2019.

[61] Keller CJ, Huang Y, Herrero JL, Fini ME, Du V, Lado FA, et al. Induction and Quantification of Excitability Changes in Human Cortical Networks. J Neurosci 2018;38:5384-98. https://doi.org/10.1523/JNEUROSCI.1088-17.2018. 
[62] Chatterjee S, Hadi AS. Influential Observations, High Leverage Points, and Outliers in Linear Regression. Statist Sci 1986;1.

https://doi.org/10.1214/ss/1177013622.

[63] N Draper, Smith H. Applied Regression Analysis. 2nd ed. Wiley; 1981.

[64] McClintock SM, Reti IM, Carpenter LL, McDonald WM, Dubin M, Taylor SF, et al. Consensus Recommendations for the Clinical Application of Repetitive Transcranial Magnetic Stimulation (rTMS) in the Treatment of Depression: (Consensus Statement). J Clin Psychiatry 2018;79:35-48. https://doi.org/10.4088/JCP.16cs10905.

[65] Fuggetta G, Fiaschi A, Manganotti P. Modulation of cortical oscillatory activities induced by varying single-pulse transcranial magnetic stimulation intensity over the left primary motor area: A combined EEG and TMS study. NeuroImage 2005;27:896-908. https://doi.org/10.1016/j.neuroimage.2005.05.013. [66] Lioumis P, Zomorrodi R, Hadas I, Daskalakis ZJ, Blumberger DM. Combined Transcranial Magnetic Stimulation and Electroencephalography of the Dorsolateral Prefrontal Cortex. JoVE 2018:57983. https://doi.org/10.3791/57983.

[67] Paus T, Sipila PK, Strafella AP. Synchronization of Neuronal Activity in the Human Primary Motor Cortex by Transcranial Magnetic Stimulation: An EEG Study. Journal of Neurophysiology 2001;86:1983-90.

https://doi.org/10.1152/jn.2001.86.4.1983.

[68] Mantell KE, Sutter EN, Shirinpour S, Nemanich ST, Lench DH, Gillick BT, et al. Evaluating transcranial magnetic stimulation (TMS) induced electric fields in pediatric stroke. NeuroImage: Clinical 2021;29:102563.

https://doi.org/10.1016/j.nicl.2021.102563.

[69] Shahin AJ, Backer KC, Rosenblum LD, Kerlin JR. Neural Mechanisms Underlying Cross-Modal Phonetic Encoding. J Neurosci 2018;38:1835-49. https://doi.org/10.1523/JNEUROSCI.1566-17.2017.

[70] Shahin AJ. Neural evidence accounting for interindividual variability of the McGurk illusion. Neuroscience Letters 2019;707:134322.

https://doi.org/10.1016/j.neulet.2019.134322.

[71] Shen S, Kerlin JR, Bortfeld H, Shahin AJ. The Cross-Modal Suppressive Role of Visual Context on Speech Intelligibility: An ERP Study. Brain Sciences 2020;10:810. https://doi.org/10.3390/brainsci10110810.

[72] Novembre G, Pawar VM, Kilintari M, Bufacchi RJ, Guo Y, Rothwell JC, et al. The effect of salient stimuli on neural oscillations, isometric force, and their coupling. NeuroImage 2019;198:221-30. https://doi.org/10.1016/j.neuroimage.2019.05.032. [73] Rogasch NC, Thomson RH, Farzan F, Fitzgibbon BM, Bailey NW, HernandezPavon JC, et al. Removing artefacts from TMS-EEG recordings using independent component analysis: Importance for assessing prefrontal and motor cortex network properties. NeuroImage 2014;101:425-39. https://doi.org/10.1016/j.neuroimage.2014.07.037.

[74] Ross JM, Iversen JR, Balasubramaniam R. Motor simulation theories of musical beat perception. Neurocase 2016;22:558-65.

https://doi.org/10.1080/13554794.2016.1242756. 
[75] Ross JM, Balasubramaniam R. Physical and neural entrainment to rhythm: human sensorimotor coordination across tasks and effector systems. Front Hum Neurosci 2014;8. https://doi.org/10.3389/fnhum.2014.00576.

[76] Ross JM, Iversen JR, Balasubramaniam R. The Role of Posterior Parietal Cortex in Beat-based Timing Perception: A Continuous Theta Burst Stimulation Study. Journal of Cognitive Neuroscience 2018;30:634-43.

https://doi.org/10.1162/jocn_a_01237.

[77] Grube M, Cooper FE, Chinnery PF, Griffiths TD. Dissociation of durationbased and beat-based auditory timing in cerebellar degeneration. Proceedings of the National Academy of Sciences 2010;107:11597-601.

https://doi.org/10.1073/pnas.0910473107.

[78] Grube M, Lee K-H, Griffiths TD, Barker AT, Woodruff PW. Transcranial magnetic theta-burst stimulation of the human cerebellum distinguishes absolute, duration-based from relative, beat-based perception of subsecond time intervals. Front Psychol 2010;1:171. https://doi.org/10.3389/fpsyg.2010.00171.

[79] Iversen JR, Balasubramaniam R. Synchronization and temporal processing. Current Opinion in Behavioral Sciences 2016;8:175-80.

https://doi.org/10.1016/j.cobeha.2016.02.027.

[80] Patel AD, Iversen JR. The evolutionary neuroscience of musical beat perception: the Action Simulation for Auditory Prediction (ASAP) hypothesis. Front Syst Neurosci 2014;8. https://doi.org/10.3389/fnsys.2014.00057.

[81] Teki S, Grube M, Griffiths TD. A Unified Model of Time Perception Accounts for Duration-Based and Beat-Based Timing Mechanisms. Front Integr Neurosci 2012;5. https://doi.org/10.3389/fnint.2011.00090.

[82] Teki S, Grube M, Kumar S, Griffiths TD. Distinct Neural Substrates of Duration-Based and Beat-Based Auditory Timing. Journal of Neuroscience 2011;31:3805-12. https://doi.org/10.1523/JNEUROSCI.5561-10.2011.

[83] Wu CC, Hamm JP, Lim VK, Kirk IJ. Musical training increases functional connectivity, but does not enhance mu suppression. Neuropsychologia 2017;104:223-33. https://doi.org/10.1016/j.neuropsychologia.2017.08.029. [84] Gordon CL, Spivey MJ, Balasubramaniam R. Corticospinal excitability during the processing of handwritten and typed words and non-words. Neuroscience Letters 2017;651:232-6. https://doi.org/10.1016/j.neulet.2017.05.021.

[85] Gordon CL, Iacoboni M, Balasubramaniam R. Multimodal Music Perception Engages Motor Prediction: A TMS Study. Front Neurosci 2018;12:736.

https://doi.org/10.3389/fnins.2018.00736. 